\title{
Proportional Concessions and the Leximin Solution*
}

\author{
Bram Driesen $^{\dagger}$
}

This version, September 2011

\begin{abstract}
In this article we introduce a new axiom for bargaining solutions, named Proportional Concession Monotonicity (PCM), which imposes that no player benefit when all players collectively make proportional concessions with respect to their respective utopia values. We reconsider the leximin solution (Imai, 1983), and obtain an alternative characterization on the basis of PCM.
\end{abstract}

JEL-Classification: $\mathrm{C} 78$

Keywords: Bargaining, axiomatic characterization, leximin solution.

\section{Introduction}

The lexicographic maxmin solution - or leximin solution - for bargaining problems is a bargaining solution based on a wealth criterion proposed by Rawls (1971) that considers most preferrable from a societal stance, the outcome that maximizes the payoffs of the worst-off individuals in the economy. The solution embodies this idea by assigning to each bargaining problem the unique outcome that results from the lexicographic optimization procedure that first maximizes the payoffs of the worst-off individuals, then the payoffs of the second worst-off individuals, then the third worst-off, and so on, until all the fruits of cooperation have been allocated.

We consider the scale-invariant variation of the leximin solution, proposed by Imai (1983), which constitutes a lexicographic extension of the wellknown Kalai-Smorodinsky bargaining solution (Kalai \& Smorodinsky, 1975). While the original leximin solution has seen a number of alternative axiomatic characterizations (Thomson \& Lensberg, 1989; Chun \& Peters, 1989; Chang \& Hwang,

* Partly written while at Óbuda University, Budapest. The author thanks the funding of the OTKA (Hungarian Fund for Scientific Research, NF-72610).

†University of Heidelberg, Department of Economics, Bergheimerstraße 58, 69115 Heidelberg, Germany. Telephone: +496221 542958. Email address: bram.driesen@awi.uniheidelberg.de. 
1999, 2001; Chen, 2000), Imai's scale invariant version has received less attention. Notable exceptions are Chang \& Liang (1998) who obtain an alternative characterization for three-person problems by weakening Imai's monotonicity axiom, and Chun \& Peters (1989) who illustrate how their characterization of the original leximin solution can be modified to one of the lexicographic KalaiSmorodinsky solution.

In this article we provide another characterization for Imai's solution, using Pareto Optimality and a new axiom named Proportional Concession Monotonicity (PCM). In the context of bargaining, a concession is defined as a player's willingness to forego feasible outcomes yielding a payoff higher than a certain utility level. The use of concessions in bargaining theory goes back as far as the work of Zeuthen (1930), and the concept has been more recently applied by a.o. Anbarci (1995) and Driesen et al. (2011). The axiom PCM introduced here, imposes that no bargainer make a strict gain when all collectively make concessions w.r.t. their respective utopia values, that are proportional in size.

\section{Preliminaries}

An $n$-person bargaining problem is fully defined by a player set $N:=\{1, \ldots, n\}$, $n \geq 2$, a feasible set $S \subset \mathbb{R}^{N}$, and a disagreement point $d \in S$. The set $\mathbb{R}^{N}$ denotes the players' payoff space in the sense that an outcome $x \in \mathbb{R}^{N}$ assigns to each player $i \in N$ a utility payoff of $x_{i}$. The interpretation is that players seek agreement on a feasible outcome $z \in S$, and that failure to cooperate results in the disagreement point $d$, which is an unfavorable outcome for all players. In general, a bargaining problem is denoted by the pair $(S, d)$. However, without loss of generality, we make the simplifying assumption that $d=\mathbf{0}$, where $\mathbf{0} \in \mathbb{R}^{N}$ denotes the vector that assigns a zero payoff to each bargainer. This allows for denoting bargaining problems by their feasible sets, i.e. writing $S$ instead of $(S, \mathbf{0})$.

For vectors $x, y \in \mathbb{R}^{N}, x>y$ denotes $x_{i}>y_{i}$ for all $i \in N, x \geqq y$ means $x_{i} \geqq y_{i}$ for all $i \in N$, and $x \geq y$ denotes $x \geqq y$ and $x \neq y$. The relations ' $<$ ', ' $\leqq$ ' and ' $\leq$ ' are defined similarly. The sets $\mathbb{R}_{+}^{N}:=\left\{z \in \mathbb{R}^{N} \mid z \geqq \mathbf{0}\right\}$ and $\mathbb{R}_{++}^{N}:=\left\{\bar{z} \in \mathbb{R}^{N} \mid z>\mathbf{0}\right\}$ define the positive resp. the strictly positive orthant of $\mathbb{R}^{N}$. We make the usual assumptions on bargaining problems $S$, that $S$ is convex and closed, that the problem is non-degenerate in the sense that there exist $z \in S$ with $z>\mathbf{0}$, and that $S \cap \mathbb{R}_{+}^{N}$, the set of individually rational outcomes, is compact. The class of all such problems is denoted $\mathcal{B}$.

A set $S \subset \mathbb{R}^{N}$ is said to be comprehensive whenever $x \in S$ and $x \geqq y$ imply that $y \in S$. Comprehensiveness of a problem $S \in \mathcal{B}$ may be interpreted as free disposal of utility. The comprehensive hull of a set $S \subset \mathbb{R}^{N}$ is defined as the smallest comprehensive set that contains $S$. It is denoted com $S$.

The vector $e(Q)$ where $Q \subseteq N$ and $Q \neq \emptyset$ denotes the outcome that assigns to all players $i \in Q$ the payoff 1 and to all players not in $Q$ the payoff 0 . The utopia point $u(S)$ of a problem $S \in \mathcal{B}$ is the outcome in $\mathbb{R}^{N}$ that accords each bargainer $i \in N$ the maximal utility payoff she can obtain from an individually 
rational outcome. That is, $u(S):=\left(u_{1}(S), \ldots, u_{n}(S)\right)$ where $u_{i}(S):=\max \left\{z_{i} \mid\right.$ $\left.z \in S \cap \mathbb{R}_{+}^{N}\right\}$. For vectors $x \in \mathbb{R}_{++}^{N}$, define $x^{-1}:=\left(1 / x_{1}, \ldots, 1 / x_{n}\right)$. For $S \in \mathcal{B}$ define the normalized problem $\bar{S}:=u(S)^{-1} S$. Clearly, $\bar{S} \in \mathcal{B}$ and $u(\bar{S})=e(N)$.

A bargaining solution, or solution, is a map $\varphi: \mathcal{B} \rightarrow \mathbb{R}^{N}$ that assigns to each problem $S \in \mathcal{B}$ an outcome $\varphi(S) \in S$. For any problem, $\varphi$ thus determines a unique feasible outcome on which the players in $N$ compromise.

\subsection{The leximin solution}

Based on Kalai's (1977) work, Imai (1983) introduces a lexicographic extension of the Kalai-Smorodinsky bargaining solution, that reconciles monotonicity considerations with efficiency.

Definition. Let $\succ^{l}$ denote the lexicographic ordering on $\mathbb{R}^{N}$. That is, $x \succ^{l} y$ if and only if $x_{i}>y_{i}$ for some $i \in N$ and $x_{j}=y_{j}$ for all $j<i$. Let $\mu$ be such that for each $x \in \mathbb{R}^{N}$ there is a permutation $\pi$ for $N$ with $\mu(x)=\pi(x)$ and $\mu_{1}(x) \leqq \ldots \leqq \mu_{n}(x)$. The lexicographic maxmin ordering $\succ$, is defined by $x \succ y$ if and only if $\mu(x) \succ^{l} \mu(y)$. For a set $S \subset \mathbb{R}^{N}$, the point $\xi(S) \in \mathbb{R}^{N}$ denotes the $\succ$-maximal point of $S$, i.e. $\xi(S) \in S$ and $\xi(S) \succ z$ for all $z \in S \backslash\{\xi(S)\}$. The leximin solution $L: \mathcal{B} \rightarrow \mathbb{R}^{N}$ (Imai, 1983) is defined by

$$
L(S):=u \xi(\bar{S})
$$

where $u:=u(S)$.

Imai shows that $L$ is found by the following procedure. Consider $S \in \mathcal{B}$. Starting from the disagreement point $\mathbf{0} \equiv x^{0}$, increase the utilities of all players simultaneously in the direction of the utopia point $u(S)$, until the boundary of the set $S$ is reached, say in the point $x^{1}$. Consider the players for whom the utilities can be further increased from $x^{1}$ without stepping out of the bargaining set. Starting from $x^{1}$, increase the utilities of these players further in the direction of their respective utopia values, again until the boundary of $S$ is reached, say in the point $x^{2}$. Continue this procedure until finally a point $x^{k}$ is reached from which no player's utility payoff can be further increased without stepping out of the bargaining set. Then $L(S)=x^{k}$.

\subsection{Axioms}

A list of properties, or axioms, is said to characterize a bargaining solution $\varphi: \mathcal{B} \rightarrow \mathbb{R}^{N}$, whenever $\varphi$ is the unique solution that satisfies these properties. The purpose of this article is to give an alternative characterization of the leximin solution. The first axiom used is Pareto Optimality, a classic property introduced by Nash (1950) that says that the entire produce of cooperation should be allocated. For $S \in \mathcal{B}$, the Pareto set $P(S):=\{z \in S \mid x \geqq z$ implies $x=z\}$ contains all points in $S$ on which there exists no feasible Pareto improvement.

$(\mathrm{PO}) \varphi: \mathcal{B} \rightarrow \mathbb{R}^{N}$ satisfies Pareto Optimality if $\varphi(S) \in P(S)$ for all $S \in \mathcal{B}$. 
Kalai (1977) introduces the axiom Monotonicity, which says that a contraction of the set of feasible allocations should not be beneficial for any bargainer.

$(\mathrm{MON}) \varphi: \mathcal{B} \rightarrow \mathbb{R}^{N}$ satisfies Monotonicity if $\varphi(S) \geqq \varphi(T)$ for all $S, T \in \mathcal{B}$ with $S \supseteq T$.

It is wellknown that $L$ violates MON. We introduce a version of this property, that is weaker in the sense that the contraction of the feasible set is of a specific type. Following Zeuthen (1930), a concession is defined as a player's willingness to forego all outcomes that yield him a utility payoff that exceeds a certain utility level. The property Proportional Concession Monotonicity says that a contraction of the set of feasible outcomes that results when all bargainers make concessions that are proportional in size, should not benefit a single player.

$(\mathrm{PCM}) \varphi: \mathcal{B} \rightarrow \mathbb{R}^{N}$ satisfies Proportional Concession Monotonicity if for all $S, T \in \mathcal{B}$ with

$$
T:=\{z \in S \mid z \leqq \gamma u(S)\}
$$

for some $\gamma>0$, we have $\varphi(S) \geqq \varphi(T)$.

Note that when outcomes are interpreted as von Neumann-Morgenstern utility payoffs, then the concessions defined in the axiom PCM are equal in normalized vNM utility terms. Thus, PCM imposes that no player gain when all players lower demands 'equally' in search of a suitable compromise.

\section{Main Result}

In the remainder of this article we prove the following Theorem.

Theorem 3.1. A bargaining solution $\varphi: \mathcal{B} \rightarrow \mathbb{R}^{N}$ satisfies PO and PCM if and only if $\varphi=L$.

The proof of Theorem 3.1 is separated in two Propositions, the first of which establishes that the solution $L$ satisfies the axioms of Theorem 3.1.

Proposition 3.2. The bargaining solution L satisfies PO and PCM.

Lemma 3.3. For all $S \subset \mathbb{R}^{N}, \xi(S)=\xi(\operatorname{com} S)$.

Proof. Let $S \subset \mathbb{R}^{N}$. If $S=\operatorname{com} S$ the result is trivial. Thus, assume $S \subset \operatorname{com} S$. Observe that either $\xi(\operatorname{com} S) \succ \xi(S)$ or $\xi(\operatorname{com} S)=\xi(S)$. Take some $x \in(\operatorname{com} S) \backslash S$. There is an $i \in N$ and $a>0$ such that $x^{\prime}:=x+a e(i) \in S$; then $x^{\prime} \succ x$. Thus, a point in $($ com $S) \backslash S$ is always $\succ$-dominated by a point in $S$. It follows that $\xi(\operatorname{com} S) \succ \xi(S)$ is impossible. Hence, $\xi(S)=\xi(\operatorname{com} S)$.

Note that Chang and Hwang (1999) already note this property of $\xi$.

Lemma 3.4. For each set $S \subset \mathbb{R}^{N}$ and constant $\beta>0$, we have $\xi(\beta S)=\beta \xi(S)$. 
Proof. Let $x, y \in S$ with $x \succ y$. Then there is an $i \in N$ such that $\mu_{j}(x)=$ $\mu_{j}(y)$ for all $j<i$ and $\mu_{i}(x)>\mu_{i}(y)$. Hence, $\beta \mu_{j}(x)=\beta \mu_{j}(y)$ for all $j<i$ and $\beta \mu_{i}(x)>\beta \mu_{i}(y)$. Since $\beta \mu_{i}(z)=\mu_{i}(\beta z)$ for each $z \in S$ and $i \in N$, this implies $\mu_{j}(\beta x)=\mu_{j}(\beta y)$ for all $j<i$ and $\mu_{i}(\beta x)>\mu_{i}(\beta y)$. That is, $\beta x \succ \beta y$. Since the implications can be reversed, it follows that $x \succ y$ if and only if $\beta x \succ \beta y$.

Let $x:=\xi(S)$. Then $x \succ y$ for all $y \in S$ with $y \neq x$. By the above, this is equivalent to $\beta x \succ \beta y$ for all $\beta y \in \beta S$ with $\beta y \neq \beta x$. This in turn, is equivalent to $\beta x=\xi(\beta S)$. It follows that $\beta \xi(S)=\xi(\beta S)$.

Proof of Proposition 3.2. It is immediate from the definition that $L$ satisfies PO. It is thus sufficient to show that $L$ satisfies PCM. Consider the problem $S \in \mathcal{B}$ with $S=\bar{S}$. From the definition of $L$ it follows that this choice is without loss of generality. Let $T \in \mathcal{B}$ with $T:=\{z \in S \mid z \leqq \gamma u(S)\}$ for some $\gamma>0$. Then $T=S \cap\left\{z \in \mathbb{R}^{N} \mid z_{i} \leqq \gamma\right.$ for all $\left.i \in N\right\}$. Consider $S^{\prime}:=$ com $S$. Let $z^{*}:=\xi\left(S^{\prime}\right)$, and construct the point $\hat{z}$ where $\hat{z}_{i}:=\min \left\{\gamma, z_{i}^{*}\right\}$ for all $i \in N$. Since $z^{*} \in S^{\prime}$ and $\hat{z} \leqq z^{*}$, it follows from comprehensiveness of $S^{\prime}$ that $\hat{z} \in S^{\prime}$. Moreover, $\hat{z} \in\left\{z \in \mathbb{R}^{N} \mid z_{i} \leqq \gamma\right.$ for all $\left.i \in N\right\}$. Define $T^{\prime}:=\operatorname{com} T$, and observe that $T^{\prime}=S^{\prime} \cap\left\{z \in \mathbb{R}^{N} \mid z_{i} \leqq \gamma\right.$ for all $\left.i \in N\right\}$. Hence, $\hat{z} \in T^{\prime}$. Suppose $\xi\left(T^{\prime}\right) \neq \hat{z}$. This implies there is a $z \in T^{\prime}$ such that $z \succ \hat{z}$. We distinguish between three cases.

1. $\gamma \leqq \mu_{1}\left(z^{*}\right)$. Then $\hat{z}=(\gamma, \ldots, \gamma)$. In that case, $z \succ \hat{z}$ implies there is an $i \in N$ such that $z_{i}>\gamma$. But then $z \notin T^{\prime}$, a contradiction.

2. $\gamma>\mu_{n}\left(z^{*}\right)$. Then $\hat{z}=z^{*}$. In that case, $z \succ \hat{z}$ implies $z \succ z^{*}$. Since $z \in T^{\prime} \subset S^{\prime}$, this implies $\xi\left(S^{\prime}\right) \neq z^{*}$, a contradiction.

3. There is a $k \in\{2, \ldots, n\}$ such that $\mu_{k-1}\left(z^{*}\right)<\gamma \leqq \mu_{k}\left(z^{*}\right)$. Note that $z \succ \hat{z}$ implies there is an $i \in\{2, \ldots, n\}$ such that $\mu_{i^{\prime}}(z)=\mu_{i^{\prime}}(\hat{z})$ for all $i^{\prime}<i$ and $\mu_{i}(z)>\mu_{i}(\hat{z})$. If $i \geqq k$, then as in argument $1, \mu_{i}(z)>\gamma$ implying $z \notin T^{\prime}$. If $i<k$, then $z \succ \hat{z}$ implies $z \succ z^{*}$, which as in argument 2 means $\xi\left(S^{\prime}\right) \neq z^{*}$. Both are contradictions.

It follows that $\xi\left(T^{\prime}\right)=\hat{z}$. That is, $\xi_{i}(\operatorname{com} T)=\min \left\{\gamma, \xi_{i}(\operatorname{com} S)\right\}$ for all $i \in N$. By Lemma 3.3 this implies $\xi_{i}(T)=\min \left\{\gamma, \xi_{i}(S)\right\}$ for all $i \in N$.

By definition of $L, L(S)=\xi(S)$. Observe that $u(T)=\gamma e(N)$, implying $\bar{T}=\frac{1}{\gamma} T$. Then by Lemma 3.4 and the definition of $L$,

$$
L(T)=u(T) \xi(\bar{T})=\gamma \xi\left(\frac{1}{\gamma} T\right)=\xi(T) .
$$

Hence, $L_{i}(T)=\min \left\{\gamma, L_{i}(S)\right\} \leqq L_{i}(S)$ for all $i \in N$. This concludes the proof.

Proposition 3.5 establishes that $L$ is the unique solution satisfying the axioms of Theorem 3.1.

Proposition 3.5. If $\varphi: \mathcal{B} \rightarrow \mathbb{R}^{N}$ satisfies $P O$ and PCM, then $\varphi=L$. 
Consider a bargaining problem $S \in B$. Following Imai (1983), we define the sequences $\left\{x^{j}\right\}_{j=0}^{k},\left\{\gamma^{j}\right\}_{j=1}^{k},\left\{\alpha^{j}\right\}_{j=1}^{k}$, and $\left\{Q^{j}\right\}_{j=1}^{k}$.

Definition. Let $V \subset \mathbb{R}^{N}$. Given $y \in \operatorname{com} V$, let

$$
Q(V, y):=\{i \in N \mid y+\varepsilon e(i) \in \operatorname{com} V \text { for some } \varepsilon>0\} .
$$

For $y \in \operatorname{com} V$ with $Q(V, y)=\emptyset$, define $\alpha(V, y):=0$ and $x(V, y):=y$. For $y \in \operatorname{com} V$ such that $Q(V, y) \neq \emptyset$, define

$$
x(V, y):=y+\alpha(V, y) e(Q(V, y))
$$

where

$$
\alpha(V, y):=\max \{\alpha \in \mathbb{R} \mid y+\alpha e(Q(V, y)) \in \operatorname{com} V\} .
$$

Let $\bar{S}$ be the normalized problem associated with $S$, and let $u:=u(S)$. Construct the sequence $\left\{x^{j}\right\}_{j=0}^{\infty}$ where $x^{0}:=\mathbf{0}$ and $x^{j}:=u x\left(\bar{S}, u^{-1} x^{j-1}\right)$ for $j \geqq 1$. Let $k$ be the smallest integer such that $x^{k}=x^{k+1}$. Then for $j=1, \ldots, k$ define

$$
Q^{j}:=Q\left(\bar{S}, u^{-1} x^{j-1}\right), \quad \alpha^{j}:=u \alpha\left(\bar{S}, u^{-1} x^{j-1}\right), \text { and } \gamma^{j}:=\sum_{j^{\prime}=1}^{j} \alpha^{j} .
$$

Note that for each $j, Q^{j}$ is equivalently defined by $Q\left(S, x^{j-1}\right)$.

A straightforward adaptation of Imai's (1983) Lemmas 3 and 4 shows that $x^{k} \in P(S)$ and that $L(S)=x^{k}$. Hence, to establish Proposition 3.5, it is sufficient to prove that any bargaining solution satisfying PO and PCM applied to the problem $S$, yields $x^{k}$. Imai's characterization of $L$ is obtained by making use of auxiliary bargaining problems. The present approach is similar. For $j=1, \ldots, k$, define

$$
T^{j}:=\left\{z \in S \mid z \leqq \gamma^{j} u\right\} .
$$

Note that $\gamma^{j}>0$ implies $T^{j} \in \mathcal{B}$ for each $j$. For $j=2, \ldots, k$ define the sets

$$
G^{j}:=P\left(T^{j}\right) \cap\left\{z \in S \mid z_{i}=x_{i}^{j-1} \text { for all } i \notin Q^{j}\right\} .
$$

The following Lemma is useful.

Lemma 3.6. $G^{j}=\left\{x^{j}\right\}$ for each $j=2, \ldots, k$.

Proof. Take some $1<j \leqq k$, and observe that $T^{j}=S \cap S^{j}$, where

$$
S^{j}:=\left\{z \in \mathbb{R}^{N} \mid z \leqq \gamma^{j} u\right\} .
$$

Note that $x^{j} \in T^{j}$. For any $i \in N$ we either have $i \notin Q\left(S, x^{j}\right)$ or $i \in Q\left(S, x^{j}\right)$. In case of the latter we have $x_{i}^{j}=\gamma^{j} u_{i}$, implying $i \notin Q\left(S^{j}, x^{j}\right)$. Hence, $Q\left(T^{j}, x^{j}\right)=$ $\emptyset$. It follows that $x^{j} \in P\left(T^{j}\right)$. By construction, $x_{i}^{j}=x_{i}^{j-1}$ for all $i \notin Q^{j}$, implying $x^{j} \in G^{j}$. 
Assume that there is a $y \in G^{j}$ with $y \neq x^{j}$. That is, $y_{i}=x_{i}^{j-1}$ for all $i \notin Q^{j}$, $y \in P\left(T^{j}\right)$, and $y \neq x^{j}$. If either $y \geqq x^{j}$ or $y \leqq x^{j}$, then $y=x^{j}$. Thus, $y \neq x^{j}$ implies there are $i^{\prime}, i^{\prime \prime} \in Q^{j}$ with $y_{i^{\prime}}<x_{i^{\prime}}^{j}$ and $y_{i^{\prime \prime}}>x_{i^{\prime \prime}}^{j}$. Since $x_{i}^{j}=\gamma^{j} u_{i}$ for all $i \in Q^{j}$, the latter implies $y \notin S^{j}$, contradicting $y \in G^{j}$. It follows that $G^{j}=\left\{x^{j}\right\}$.

Proof of Proposition 3.5. Let $\varphi: \mathcal{B} \rightarrow \mathbb{R}^{N}$ be a bargaining solution satisfying PO and SPC. Observe that $x^{1} \in T^{1} \subseteq \operatorname{com}\left\{x^{1}\right\}$, implying $P\left(T^{1}\right)=\left\{x^{1}\right\}$. By PO it follows that $\varphi\left(T^{1}\right)=x^{1}$. For $1<j \leqq k$, assume that $\varphi\left(T^{j-1}\right)=x^{j-1}$. Observe that

$$
\begin{aligned}
T^{j-1} & =\left\{z \in S \mid z \leqq \gamma^{j-1} u(S)\right\} \\
& =\left\{z \in T^{j} \mid z \leqq \gamma^{j-1} u(S)\right\} \\
& =\left\{z \in T^{j} \mid z \leqq\left(\gamma^{j-1} / \gamma^{j}\right) u\left(T^{j}\right)\right\},
\end{aligned}
$$

and that $\gamma^{j-1} / \gamma^{j}>0$. Then by PCM we obtain $\varphi\left(T^{j}\right) \geqq \varphi\left(T^{j-1}\right)$. Observe that for any $x \in T^{j}$ with $x \geqq x^{j-1}$, we have $x_{i}=x_{i}^{j-1}$ for all $i \notin Q^{j}$. It follows that $\varphi_{i}\left(T^{j}\right)=x_{i}^{j-1}$ for all $i \notin Q^{j}$. By PO we have $\varphi\left(T^{j}\right) \in P\left(T^{j}\right)$. Hence, $\varphi\left(T^{j}\right) \in G^{j}$. By Lemma 3.6 this implies $\varphi\left(T^{j}\right)=x^{j}$. It follows that $\varphi\left(T^{k}\right)=x^{k}$. Since $T^{k}=\left\{z \in S \mid z \leqq \gamma^{k} u\right\}$, it follows by PCM that $\varphi(S) \geqq \varphi\left(T^{k}\right)$, and thus, by the above argument, $\varphi(S) \geqq x^{k}$. By construction, $x^{k} \in P(S)$. Hence, $\varphi(S)=x^{k}$. This concludes the proof.

For $S \in \mathcal{B}$ the Nash bargaining solution yields the unique point $x^{*}$ in $S$ that maximizes the function $\prod_{i \in N} x_{i}$. It satisfies PO but violates PCM.

Example. For $n=2$, consider the problem $S \in \mathcal{B}$ with

$$
S:=\operatorname{com}\{e(1), e(2),(1 / 2,3 / 4)\} .
$$

It is easily checked that the Nash bargaining solution of this game is given by $(1 / 2,3 / 4)$. Consider next the problem $\hat{S}$ where $\hat{S}:=\left\{z \in S \mid z_{i} \leqq 2 / 3\right.$ for $i=$ $1,2\}$, and observe that the Nash solution now yields the outcome $(5 / 9,2 / 3)$. Since $5 / 9>1 / 2$, we obtain a violation of PCM.

The solution $D: \mathcal{B} \rightarrow \mathbb{R}^{N}$, defined by $D(S):=\mathbf{0}$ for all $S \in \mathcal{B}$, satisfies PCM but not PO. It follows from these two observations that the axioms PCM and $\mathrm{PO}$ are independent.

\section{Final Remarks}

The leximin solution involves a normalization of the bargaining problem w.r.t. the players' utopia values. An alternative version is obtained when the problem is normalized not w.r.t. the players' utopia values, but their global utopia values. Imai (1983) points out that his characterization proof can be modified in a 
straightforward way, to also characterize this alternative solution. The same is true for the characterization proof presented in this article. In particular, it is sufficient to replace the utopia point $u(S)$ in the axiom PCM by the problem's global utopia point.

Chun \& Peters (1991) define and characterize the lexicographic equal-loss solution. ${ }^{1}$ Using an approach similar to the one presented in this article, it is possible to obtain an alternative characterization for this bargaining solution as well. This requires monotonicity under equal concessions, rather than proportional concessions. Moreover, it requires adding the axiom Independence of Irrelevant Alternatives other than the Ideal Point (Roth, 1977), which imposes that the solution remain invariant under contractions of the feasible set as well as changes of disagreement point, that leave the problem's utopia point unaltered. ${ }^{2}$

\section{References}

[1] Anbarci N (1995). Reference functions and balanced concessions in bargaining. The Canadian Journal of Economics, 28(3):675-682.

[2] Chang C, Liang M (1998). A characterization of the lexicographic KalaiSmorodinsky solution for $n=3$. Mathematical Social Sciences, 35(3):307319.

[3] Chang C, Hwang Y (1999). A characterization of the leximin solution of the bargaining problem. Mathematical Methods of Operations Research, 49(3):395-400.

[4] Chang C, Hwang Y (2001). Two characterizations of the lexicographic Egalitarian solution. International Game Theory Review, 3(4):315-323.

[5] Chen M (2000). Individual monotonicity and the leximin solution. Economic Theory, 15(2):353-365.

[6] Chun Y, Peters H (1991). The lexicographic equal-loss solution. Mathematical Social Sciences, 22(2):151-161.

[7] Chun Y, Peters H (1989). The lexicographic Egalitarian solution. Cahiers du C.E.R.O, 30(2):149-156.

[8] Driesen B, Perea A, Peters H (2011). The Kalai-Smorodinsky bargaining solution with loss aversion. Mathematical Social Sciences, 61(1):58-64.

\footnotetext{
${ }^{1}$ For this solution the assumption $d=\mathbf{0}$ is not without loss of generality, and needs to be relaxed.

${ }^{2}$ While it carries the same name, this is a stronger property than the one Imai (1983) employs in his characterization of the leximin solution. Imai's axiom is also introduced by Roth (1977), but there given the name Independence of Irrelevant Alternatives other than the Disagreement Point and the Ideal Point.
} 
[9] Imai H (1983). Individual monotonicity and lexicographic maxmin solution. Econometrica, 51(2):389-401.

[10] Kalai E (1977). Proportional solutions to bargaining situations: interpersonal utility comparisons. Econometrica, 45(7):1623-1630.

[11] Kalai E, Smorodinsky M (1975). Other solutions to Nash's bargaining problem. Econometrica, 43(3):513-518.

[12] Nash J (1950). The bargaining problem. Econometrica, 18(2):155-162.

[13] Rawls J (1971). A theory of justice. Harvard Univ. Press, Cambridge, Massachusetts.

[14] Roth A (1977). Independence of irrelevant alternatives, and solutions to Nash's bargaining problem. Journal of Economic Theory, 16(2):247-251.

[15] Thomson W, Lensberg T (1989). Axiomatic theory of bargaining with a variable number of agents. Cambridge Univ. Press, Cambridge.

[16] Zeuthen F (1930). Problems of monopoly and economic warfare. G Routledge \& sons, ltd, London. 